\title{
La creatividad en la formación del arquitecto, el proceso creativo y las neurociencias
}

\author{
Creativity in the Training of the Architect, the Creative Process and \\ Neurosciences
}

\section{Criatividade no treinamento de arquitetos, processo criativo e neurociência}

\author{
Miguel Navarro Velázquez \\ Universidad de Sonora, Escuela de Arquitectura, México \\ arqmnavarrov@hotmail.com \\ https://orcid.org/0000-0003-0861-0586
}

\section{Resumen}

El presente artículo expone resultados primarios sobre el papel que juega la creatividad en la arquitectura y la formación de los arquitectos, la cual es fundamental en el proceso del diseño arquitectónico, desde todo el proceso formativo en la universidad hasta la etapa profesionallaboral como egresados. Esta investigación se apoya en algunas nociones de las neurociencias, que ayudaron a entender el proceso del pensamiento creativo, crítico y analítico. Estos conocimientos ratificaron la importancia de ejercitar la creatividad, de dominarla, manipularla y controlarla para solucionar problemas arquitectónicos. Mediante la observación, el estudio de casos, las encuestas, las entrevistas y el estudio del nuevo diseño curricular de la Escuela de Arquitectura de la Universidad de Sonora (2018), se obtuvieron las variables necesarias para poder ofrecer una alternativa de solución al problema de introducir la creatividad dentro del proceso de formación del arquitecto en dicho recinto académico. Los resultados muestran que el proceso de formación de los estudiantes de arquitectura es reproductivo y no se le da la importancia que merece a la creatividad en los procesos productivos de innovación en el plan de estudios. Además, si bien la planta académica ve a la creatividad como un factor importante, no la promueve y no la incorpora 


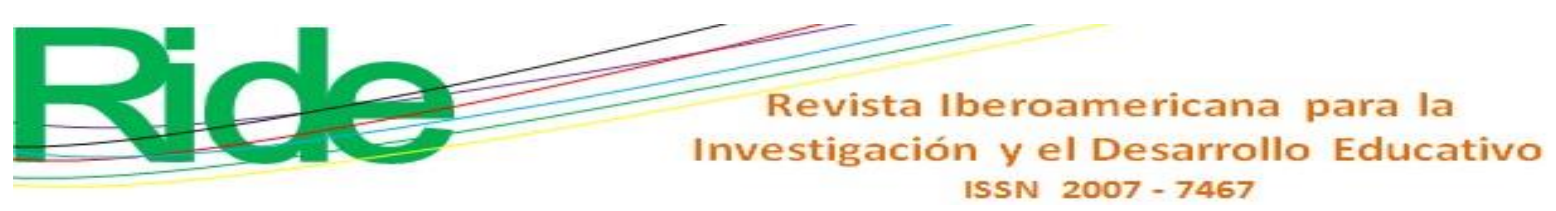

en sus actividades docentes de forma específica. Para solucionar esta situación, se propone la aplicación de una metodología epistemológica que ayude tanto en el macro como en el microdiseño curricular, que contemple incluir la creatividad como necesaria en el perfil de ingreso de los aspirantes y que le dé mayor énfasis durante el proceso de aprendizaje formativo, lo cual es imprescindible para desarrollar esta competencia, así como modificar el perfil de egreso de los futuros arquitectos.

Palabras claves: creatividad, diseño curricular, espacio arquitectónico, estudiante de arquitectura, neurociencias.

\begin{abstract}
This research was supported with some knowledge of neurosciences, which helped to understand the process of creative, critical and analytical thinking. This knowledge confirmed the importance of exercising creativity, of mastering, manipulating and controlling it to solve architectural problems. Through observation, case studies, surveys, interviews and the study of the new curricular design of the Escuela de Arquitectura at the Universidad de Sonora (2018), the necessary research variables were obtained in order to offer an alternative solution to the problem of introducing creativity into the architect's training process in the aforementioned academic precinct. The results of the research show that the process of training of architecture students is reproductive and it is not given the importance of creativity in the productive processes of innovation in the curriculum. In addition, although the academic staff sees creativity as an important factor, does not promote and does not incorporate it in their teaching activities specifically. To solve this situation, the application of an epistemological methodology was proposed that helps both the macro and the micro curricular design, that includes creativity as necessary in the profile of entry of the applicants and that is emphasized during the process of formative learning, which is essential to develop this competence, as well as modify the graduation profile of future architects.
\end{abstract}

Keywords: creativity, curriculum design, architectural space, architecture student, neuroscience. 


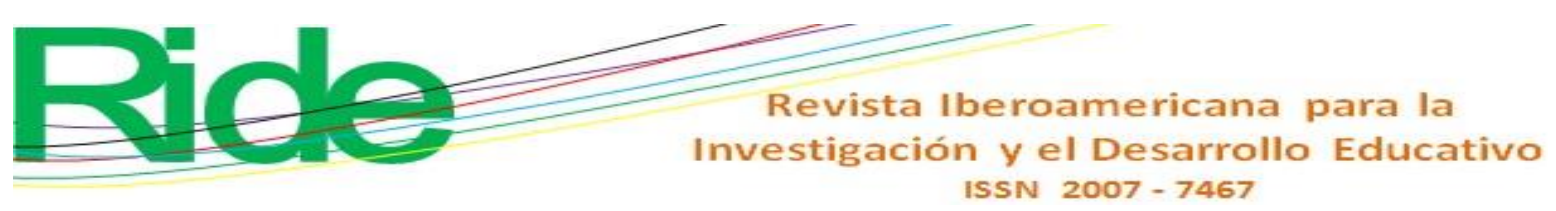

\section{Resumo}

Este artigo apresenta resultados primários sobre o papel que a criatividade desempenha na arquitetura e no treinamento de arquitetos, fundamental no processo de projeto arquitetônico, desde todo o processo de treinamento na universidade até a etapa do trabalho profissional como graduados. . Esta pesquisa é apoiada por algumas noções de neurociência, que ajudaram a entender o processo de pensamento criativo, crítico e analítico. Esse conhecimento confirmou a importância de exercitar a criatividade, dominá-la, manipulá-la e controlá-la para resolver problemas arquitetônicos. Através da observação, estudos de caso, pesquisas, entrevistas e o estudo do novo desenho curricular da Escola de Arquitetura da Universidade de Sonora (2018), foram obtidas as variáveis necessárias para poder oferecer uma solução alternativa ao problema de introduzir criatividade no processo de treinamento do arquiteto no referido recinto acadêmico. Os resultados mostram que o processo de formação dos estudantes de arquitetura é reprodutivo e a criatividade não recebe a importância que merece nos processos produtivos de inovação no currículo. Além disso, embora a equipe acadêmica veja a criatividade como um fator importante, ela não a promove e não a incorpora especificamente em suas atividades de ensino. Para resolver essa situação, propõe-se a aplicação de uma metodologia epistemológica que ajude tanto na macro quanto na microdesign do currículo, que contemple a inclusão da criatividade necessária no perfil de entrada dos candidatos e que dê maior ênfase durante o processo de aprendizagem formativa, essencial para desenvolver essa competência, bem como modificar o perfil de graduação dos futuros arquitetos.

Palavras-chave: criatividade, desenho curricular, espaço arquitetônico, estudante de arquitetura, neurociência.

Fecha Recepción: Diciembre 2019

Fecha Aceptación: Mayo 2020 


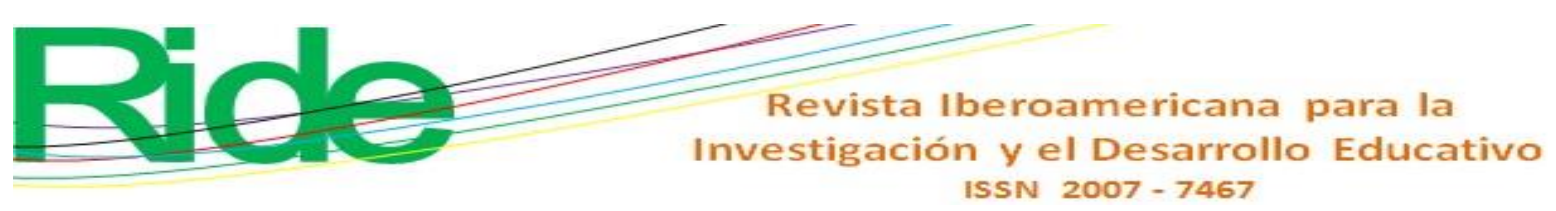

\section{Introducción}

El proceso creativo en el diseño arquitectónico es la piedra angular del trabajo de los arquitectos. Es por eso por lo que debemos dominarlo y enseñarlo de manera adecuada: convertirlo en una competencia de nuestros estudiantes. Para el desarrollo de la creatividad el cerebro del arquitecto debe tener la habilidad de identificar patrones y mostrar curiosidad por lo novedoso y por lo desconocido. Sin duda, arquitectura y creatividad son dos palabras que van de la mano, no es posible concebir una sin la otra; levantar muros y colocar techos no es hacer arquitectura.

Existen muchas y variadas definiciones de arquitectura: desde las más técnicas hasta las más poéticas. Aquí podemos definirla como la disciplina (considerada arte por algunos) que concibe espacios destinados para un uso determinado y que no solo es capaz de satisfacer las necesidades de los usuarios, sino también de brindar sensaciones. Para poder lograr esto se requiere de conocimientos teóricos, prácticos y de mucha creatividad.

Por si lo anterior no fuera suficiente, Ibo Bonilla (s. f.) realizó una recopilación de pensamientos sobre lo que es la arquitectura. A continuación, se presentan algunas de estas ideas.

En primer lugar, Luis Barragán (citado en Bonilla, s. f.) mencionó lo siguiente:

El espacio ideal debe contener en sí elementos de magia, serenidad, embrujo y misterio. Creo que estos pueden inspirar la mente de los hombres. La arquitectura es arte cuando consciente o inconscientemente se crea una atmósfera de emoción estética y cuando el ambiente suscita una sensación de bienestar (p. 9).

Tadao Ando (citado en Bonilla, s. f.), por su parte, indica lo puesto a continuación:

La arquitectura debe ser el resultado de un encuentro entre el razonamiento lógico y la creación que resulta del uso de los sentidos. No alcanza solo con el conocimiento y la respuesta lógica al programa, pero tampoco con mera sensibilidad es posible satisfacer las demandas que nos hace la realidad (p. 7).

Por último, el propio Ibo Bonilla (2004) creó su definición: “Arquitectura es esculpir el espacio para satisfacer necesidades físicas, emocionales y espirituales, protegiendo el resultado con una piel armónica con la estética, técnicas y sitio, del momento en que se realiza. (...) Es modelar metapiel social con arte" (p. 1). Como se puede observar, algunas de estas definiciones consideran a la arquitectura como un arte, porque así ha sido establecido 


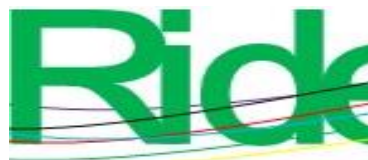

Revista Iberoamericana para la
Investigación y el Desarrollo Educativo
ISSN $2007-7467$

por mucho tiempo. Sin duda habrá algunos teóricos que difieran, sin embargo, esta discusión no es relevante para el presente trabajo, lo que se pretende es aclarar que sin creatividad no existiría el arte y la arquitectura (Ando, Heneghan y Pare, 1996).

La creatividad no figura como una de las competencias a desarrollar en el plan de estudios de la Escuela de Arquitectura de la Universidad de Sonora, lo cual sugiere dos escenarios: o es percibida como una característica inherente al programa, o se considera innecesaria.

Debido a la importancia que tiene la creatividad en el quehacer del arquitecto, debe ser considerada como una competencia a obtener y desarrollar, y para lograr esto es necesario incluirla tanto en el plan de estudios como en el perfil de egreso de los estudiantes. En muchas ocasiones, no obstante, no se tiene conciencia de esto, lo que pudiera causar la pérdida o la atrofia de la creatividad durante el proceso formativo. Lo peor es cuando los profesores ni siquiera le dan importancia y pretenden que aparezca por generación espontánea, en el mejor de los casos, o se desdeña la necesidad de tenerla, pretendiendo que con base en datos y ejercicios el estudiante resolverá los problemas espaciales creativamente. Esto no es posible.

En algunos casos se pretende utilizar un software de graficación arquitectónica para realizar el proceso de diseñar los espacios arquitectónicos, lo cual, aquí no cabe duda, es incorrecto, porque esos softwares son para dibujar, para graficar, no para diseñar, porque la máquina no piensa, sino que sigue directrices establecidas, las cuales, si bien ayudan, no sustituyen el pensamiento creativo. En la arquitectura, el pensamiento creativo requiere de sensibilidad, de involucrar los sentimientos, para poder crear espacios que ayuden al usuario a sentirse cómodo, seguro, relajado, confortable, en fin, que ayuden a que los espacios creados sirvan para lo que fueron diseñados: que nos hagan sentir, y esto precisamente es lo que propicia la innovación, el romper paradigmas espaciales y temporales (Corral y Díaz, 2009; López de Asiain, 2005; Mendoza, Zacarías y Moreno, 2015; Rueda, 2014)

El arquitecto, para realizar su profesión, deberá ser un excelente investigador, porque requiere contar con los datos necesarios para poder resolver cualquier problema espacial que se le encomiende. Pero no basta con ello, la creatividad es lo que hace la diferencia: es la que aporta los elementos especiales que diferencian a un diseño de otro, a un arquitecto de otro, a una reproducción de una innovación. 


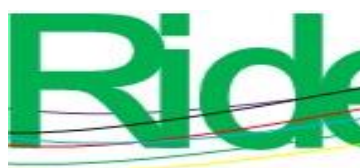

Revista Iberoamericana para la Investigación y el Desarrollo Educativo ISSN $2007-7467$

En el proceso creativo es donde se vuelven reales los sueños, donde, además de la funcionalidad remarcada en el modernismo de la Bauhaus creada por Walter Gropius en 1919, la máquina de habitar, como decía Le Corbusier, o la estética de las diferentes épocas, deberemos marcar la diferencia al unir funcionalidad, estética, sustentabilidad y creatividad para hacer arquitectura, al igual que tomar en consideración, por un lado, al usuario y considerar, por el otro, la importancia del estudio del terreno, la flora, la fauna, la zona, la accesibilidad, la infraestructura necesaria, los servicios públicos requeridos, el clima, los costos, las leyes, normas y reglamentos. Obviamente deberá tomarse en cuenta de forma especial la protección de la ecología, la sustentabilidad y la sostenibilidad, estos tres últimos son tratados generalmente como algo aparte, y no debe ser así, como si se pudiera hacer una arquitectura ecológica, sostenible y sustentable y otra no, una buena arquitectura y otra mala. Aquí, por el contrario, se afirma que existe una sola. Y para que pueda ser considerada como tal, debe reunir e integrar todos estos estudios, requisitos y cuidados; de no ser así, si bien se estaría satisfaciendo al usuario o cliente, se estaría atentando contra la sociedad, contra nuestro planeta y la humanidad: esto no podría llamarse arquitectura.

Las sensaciones son parte importante del desarrollo del ser humano y la arquitectura deberá sumar para alcanzarlo. La creatividad es la encargada de hacer sentir la arquitectura.

\section{La creatividad}

En la Enciclopedia de Psicopedagogía Océano (1998, citada en Esquivias, 2004) se define a la creatividad como la "disposición a crear que existe en estado potencial en todo individuo y a todas las edades" (p. 3). Por otra parte, en el Diccionario de las Ciencias de la Educación Santillana (1995, citado en Esquivias, 2004) se señala que el término creatividad significa "innovación valiosa y es de reciente creación” (p. 3).

Esquivias (2004), asimismo, señala lo siguiente:

El proceso creativo es una de las potencialidades más elevadas y complejas de los seres humanos, este implica habilidades del pensamiento que permiten integrar los procesos cognitivos menos complicados, hasta los conocidos como superiores para el logro de una idea o pensamiento nuevo (p. 3).

Para los fines de este trabajo definiremos a la creatividad como la capacidad del ser humano de aportar nuevas ideas, tecnologías, o expresiones artísticas, de innovar. 


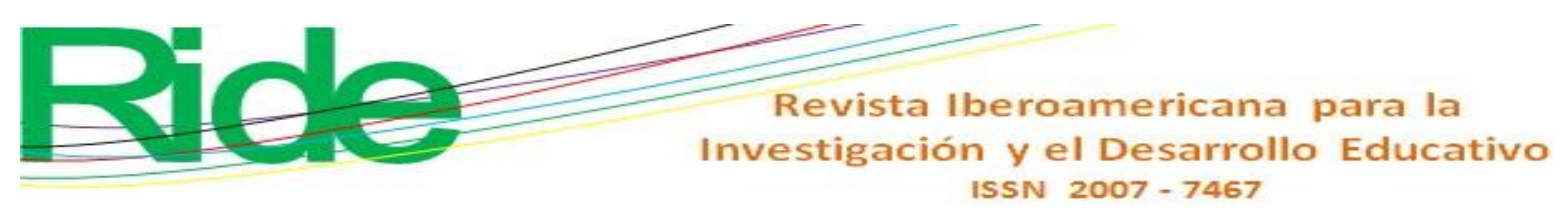

\section{Diseño arquitectónico}

Los jóvenes aspirantes a estudiar arquitectura deberán tomar conciencia de la importancia que el diseño arquitectónico tiene en su desarrollo profesional. De hecho, tal es su importancia que sin este no existirían obras de arquitectura. El diseño arquitectónico es plasmar primero con bocetos, después con planos en una dimensión, en dos dimensiones con perspectivas, renders o cualquier otra expresión gráfica arquitectónica, el proyecto en sus diferentes etapas, y las maquetas que nos muestran en tres dimensiones un proyecto que nació de una necesidad espacial, de un problema arquitectónico y se resolvió de forma profesional. Todo inicia con un problema a resolver. De ahí nace una primera idea y, en ese momento, el cerebro manda una orden a la mano para que la grafique, la exprese, la dibuje. Es por eso por lo que la graficación, el dibujo arquitectónico, es el lenguaje del arquitecto y debemos dominarlo para poder expresar de forma visible y adecuada nuestras ideas, nuestras propuestas arquitectónicas. Para lograr esto se requiere de práctica, mucha práctica, y de seguir una metodología adecuada.

\section{El proceso creativo}

¿Qué función tiene el cerebro dentro del proceso creativo? La parte derecha del cerebro es creativa, mientras que la izquierda es lógica. El hemisferio derecho está especializado en sensaciones, sentimientos y habilidades especiales visuales y sonoras, como la música o el arte, pero no verbales (la percepción del espacio, el ritmo, el color, la dimensión, la imaginación y las ensoñaciones, entre otras). El hemisferio izquierdo se ocupa de la parte verbal y la numérica (la aritmética, la secuencial, la lógica y la analítica).

"Lo fundamental es contar con la habilidad necesaria para utilizar ambos hemisferios, ya que así se está en un completo balance y se logra una creatividad más efectiva" (Alcaraz, 2006, p. 11).

Cerda (2000), por su parte, dice que la habilidad de nuestro cerebro para identificar patrones y mostrar curiosidad por lo novedoso facilita el aprendizaje, que está basado, en parte, en la capacidad creativa. Y es este pensamiento creativo, que complementa al pensamiento crítico o analítico que utiliza una metodología científica rigurosa y lineal, el que nos permite resolver una gran variedad de problemas de una forma, a veces, imprevisible. Dicho resultado es el que muchas de las veces nos ayuda a lograr una obra de arquitectura adecuada y, en más de una ocasión, una obra única por su creatividad y representatividad, 


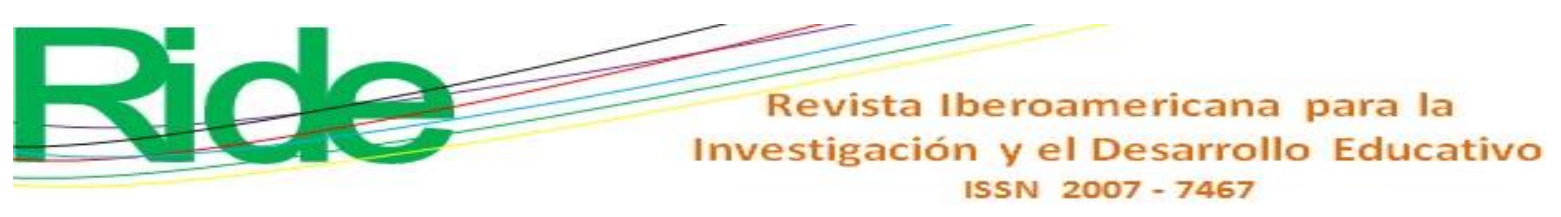

rompiendo paradigmas e implantando alguna tendencia innovadora en su tiempo y lugar (Cerda, 2000).

\section{Neurociencias}

La neurociencia es el conjunto de disciplinas científicas que estudia el sistema nervioso con el fin de acercarse a la comprensión de los mecanismos que regulan el control de las reacciones nerviosas y del comportamiento del cerebro. Disciplinas como la neuroanatomía, neurofisiología, neurofarmacología, neuroquímica, etc. Es por ello por lo que la neurociencia debe ser estudiada de manera integrada y complementaria con el fin de comprender la complejidad del cerebro (De la Barrera y Donolo, 2009)

El nuevo paradigma que se plantea en el siglo XXI en relación con la arquitectura surge con los descubrimientos de las neurociencias y los estudios de la psicología ambiental: estos avances científicos han demostrado que determinados espacios ayudan en la edad adulta a la producción de nuevas células nerviosas, y que el diseño de los espacios arquitectónicos influye en los estados emocionales y el comportamiento de los individuos (Elizondo y Rivera, 2017; Franch y Camacho, 2016). Las neurociencias y la psicología ambiental están permitiendo dar respuesta a preguntas del tipo:

- ¿ ¿Cómo afecta a nuestra conducta el grado de privacidad que nos brinda el lugar de trabajo?

- ¿Cómo interpreta nuestro cerebro el espacio en que vivimos?

- ¿Por qué los enfermos se recuperan más rápidamente en hospitales con espacios naturales y amplios ventanales al exterior?

- ¿ ¿Cómo influye la luz natural en nuestro rendimiento

- ¿Qué ambientes generan más descontento y agresión?

- ¿ ¿Qué entornos favorecen nuestra colaboración en el trabajo?

- ¿Por qué determinados espacios nos inspiran sensación de bienestar?

- ¿Cómo afectan los espacios a nuestros pensamientos o a nuestro grado de estrés?

Todos estos aspectos y muchos más son los que contempla el nuevo paradigma de la neuroarquitectura, una ciencia que acaba de nacer y que en el futuro será materia obligada en los estudios de arquitectura. Las primeras instituciones centradas en la investigación de esta nueva ciencia son relativamente recientes. Así ,en 2003, se creó en San Diego (California) la 


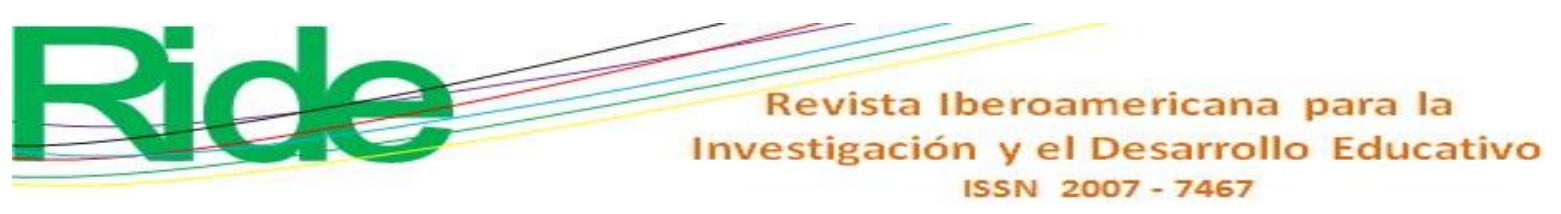

Academia de Neurociencias para la Arquitectura. Este centro tiene como misión investigar cómo debe ser el diseño del espacio en el siglo XXI para mejorar nuestro bienestar, aumentar el rendimiento y reducir la fatiga y el estrés de las ciudades.

\section{Neuroarquitectura}

Al respecto de esta disciplina, Punset (s. f.) refiere lo siguiente:

Empieza a arrojar indicios interesantes para ayudarnos a comprender cómo el hábitat en el que vivimos afecta a nuestra salud física y mental. No se trata solo de intuir que el color o el espacio tienen un impacto sobre nuestro estado de ánimo. Se trata de ir un paso más allá e indagar sobre qué efecto específico tienen los espacios sobre el estrés, las hormonas y el tipo de pensamientos que generamos (párr. 2).

Actualmente se está investigando la relación entre espacios amplios y pensamiento creativo; sobre el poder misterioso de la naturaleza para estimular tanto la concentración como la curación de las personas tras una enfermedad; o sobre el impacto de los edificios y muebles con ángulos afilados sobre la amígdala implicada en los procesos de defensa y agresión del cerebro (Gutiérrez, 2017). La neuroarquitectura debe ser tomada por los planes de estudio para los futuros arquitectos como fundamental para el logro de la creación de los espacios adecuados para el ser humano a razón de la importancia que tiene su aportación para nuestro desarrollo profesional (Elizondo y Rivera, 2017).

Como podemos apreciar, las neurociencias merecen un estudio aparte por su gran importancia en el desarrollo humano. Entre ellas, para el asunto que aquí interesa, la neuroarquitectura, que nos ayuda a entender el proceso creativo arquitectónico y el cómo podemos recurrir a él de forma consciente y eficaz (Portellano, 2000). Existen dos estrategias generales que nos permiten resolver problemas de forma diferente: una analítica, que conlleva una evaluación sistemática de las diferentes etapas en las que se puede desglosar el problema, y otra más creativa, que conlleva un proceso conocido como insight, el cual nos permite resolver el problema de forma repentina sin ser conscientes del proceso y que nos provoca gran satisfacción (Tirapu y Luna, 2008). Entender los mecanismos neurales que conlleva el insight tiene importantes implicaciones educativas debido a la relación directa que tiene este fenómeno cognitivo con el aprendizaje, la creatividad y las estrategias en la resolución de problemas (Kolb y Whishaw, 2006). 


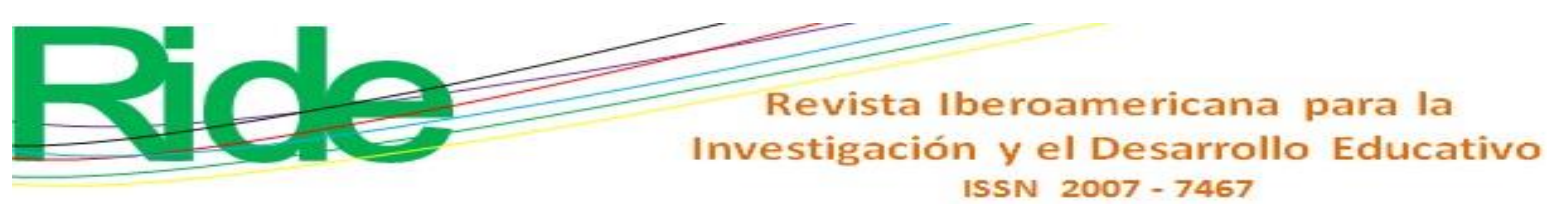

este fin. Coincido, eso sí, en darle un papel preponderante a la creatividad y enfatizar su importancia, y la necesidad de desarrollar el pensamiento creativo, crítico y analítico, que es fundamental para el arquitecto y deberá usarse como herramienta valiosa en el proceso del diseño arquitectónico, servirá como guía de acción, utilizando tanto la posición metodológica como la epistemológica para la solución de problemáticas. Además, deberá contarse con una metodología adecuada y con una visión holística del conflicto y así, a través de la metodología, ir resolviendo por partes el problema arquitectónico (Huerta, 16 de febrero de 2016; McLaren, Huerta y Rodríguez, 2010)

En este trabajo se presentan resultados sobre la creatividad en el proceso de formación del arquitecto en la Universidad de Sonora y su influencia en su desempeño laboral profesional.

\section{Método}

Utilizamos para esta investigación el método empírico de la observación, revisión de documentos, encuestas y entrevistas. Para el procesamiento de los datos obtenidos y para analizar e interpretar la información obtenida y validar los resultados de la investigación, nos apoyamos en los niveles matemático y estadístico. Según la estadística de Recursos Humanos y Servicios Estudiantiles, el Departamento de Arquitectura y Diseño de la Universidad de Sonora cuenta con una población de 61 profesores y 884 estudiantes (agosto del 2019), y está conformado por un director de división, un jefe de departamento y un coordinador. El plan de estudio es de 10 semestres y tiene como columna vertebral el Taller de Arquitectura, que se cursa en todos los semestres.

\section{Revisión y observación}

Se realizó la revisión del nuevo plan de estudio de la Escuela de Arquitectura de la Universidad de Sonora (2018), basado en competencias, con el objetivo de conocer el papel de la creatividad en el proceso de formación del arquitecto tanto en el macrodiseño como en el microdiseño curricular. Se revisó el perfil de ingreso, el modelo de actuación del profesional que contiene el perfil de egreso, los objetivos y las competencias. También se revisó el contenido de las diferentes unidades de aprendizaje, el sistema de evaluación y las indicaciones metodológicas. 


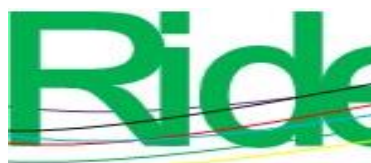

Revista Iberoamericana para la Investigación y el Desarrollo Educativo ISSN $2007-7467$

Se observó la manera en que los estudiantes trabajan para solucionar un proyecto arquitectónico, con o sin una metodología, con o sin una correcta guía de parte de sus profesores; también si el estudiante aplica los conocimientos y habilidades obtenidos en las demás disciplinas y qué actitud tiene en el proceso; en resumen, si ha ido adquiriendo las competencias requeridas y necesarias para realizar correctamente su trabajo creativo. Aunado a lo anterior, se realizó una observación general de la infraestructura que tiene la Escuela de Arquitectura para la formación de los estudiantes, espacios docentes y áreas recreativas, áreas de investigación, talleres y aulas especializadas. También, la página de internet y el laboratorio Técnicas de la Comunicación e Informática (TIC), incluyendo softwares especializados.

\section{Estudio de casos}

Mediante el estudio de los trabajos de los alumnos de los diez semestres, se revisaron y analizaron propuestas arquitectónicas, la aplicación de una metodología y sobre todo se verificó la utilización de la creatividad en sus propuestas.

\section{Encuestas}

Se realizaron encuesta a estudiantes, maestros y egresados con el propósito de conocer qué tan conscientes están del papel tan importante que tiene la creatividad en su trabajo arquitectónico.

Se realizaron encuestas a 200 alumnos de la Universidad de Sonora, del $1 .^{\circ}$ al $10 .^{\circ}$ semestre, con el propósito de conocer su punto de vista acerca de la importancia que tiene la creatividad en su preparación profesional. También a 20 maestros y a 20 arquitectos egresados de la Escuela de Arquitectura de la Universidad de Sonora.

\section{Preguntas a los estudiantes}

1) ¿La arquitectura es un arte?

2) ¿Consideras la creatividad fundamental para el arquitecto?

3) ¿Te consideras creativo?

4) ¿Sigues una metodología en tu proceso de diseño?

5) ¿Tienes alguna clase específica de creatividad?

6) ¿Te han realizado alguna prueba específica de creatividad? 


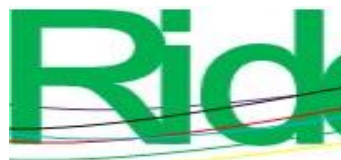

\section{Revista Iberoamericana para la Investigación y el Desarrollo Educativo ISSN $2007-7467$}

7) ¿La escuela te ha motivado expresamente a desarrollar tu creatividad con alguna actividad extracurricular?

8) ¿Los maestros te han motivado expresamente a desarrollar tu creatividad?

9) ¿La creatividad es innata?

10) ¿Consideras creativo diseñar, dibujar y graficar a mano?

11) ¿Diseñas a mano?

12) ¿Diseñas directamente en la computadora?

13) ¿Diseñar en la computadora es creativo?

14) ¿Diseñas a mano y dibujas en la computadora?

15) ¿Crees que los renders venden la arquitectura?

16) ¿Las tecnologías constructivas requieren de la creatividad?

17) ¿Las tecnologías digitales requieren de la creatividad?

18) ¿Construir requiere de la creatividad?

19) ¿La gestión requiere creatividad?

20) ¿Se requiere creatividad para elegir las estrategias de diseño?

Figura 2. Encuesta a los estudiantes

\begin{tabular}{|c|c|c|c|c|c|c|c|c|c|c|c|c|c|c|c|c|c|c|c|c|}
\hline$\%$ & 1 & 2 & 3 & 4 & 5 & 6 & 7 & 8 & 9 & 10 & 11 & 12 & 13 & 14 & 15 & 16 & 17 & 18 & 19 & 20 \\
\hline $\begin{array}{l}10 \\
0\end{array}$ & & & & & & & & & & & & & & & & & & & & \\
\hline 95 & & & & & & & & & & & & & & & & & & & & \\
\hline 90 & & & & & & & & & & & & & & & & & & & & \\
\hline 85 & & & & & & & & & & & & & & & & & & & & \\
\hline 80 & & & & & & & & & & & & & & & & & & & & \\
\hline 75 & & & & & & & & & & & & & & & & & & & & \\
\hline 70 & & & & & & & & & & & & & & & & & & & & \\
\hline 65 & & & & & & & & & & & & & & & & & & & & \\
\hline 60 & & & & & & & & & & & & & & & & & & & & \\
\hline 55 & & & & & & & & & & & & & & & & & & & & \\
\hline 50 & & & & & & & & & & & & & & & & & & & & \\
\hline 45 & & & & & & & & & & & & & & & & & & & & \\
\hline 40 & & & & & & & & & & & & & & & & & & & & \\
\hline 35 & & & & & & & & & & & & & & & & & & & & \\
\hline 30 & & & & & & & & & & & & & & & & & & & & \\
\hline 25 & & & & & & & & & & & & & & & & & & & & \\
\hline 20 & & & & & & & & & & & & & & & & & & & & \\
\hline 15 & & & & & & & & & & & & & & & & & & & & \\
\hline 10 & & & & & & & & & & & & & & & & & & & & \\
\hline 5 & & & & & & & & & & & & & & & & & & & & \\
\hline 0 & & & & & & & & & & & & & & & & & & & & \\
\hline
\end{tabular}

Fuente: Elaboración propia 


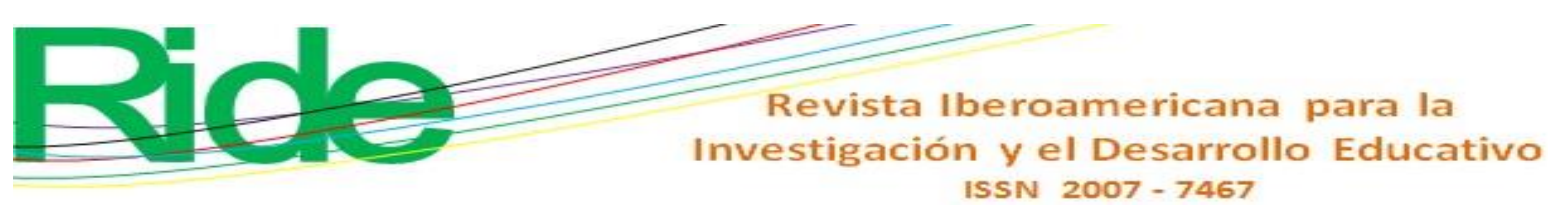

\section{Preguntas a los profesores}

1) ¿La arquitectura es un arte?

2) ¿Consideras la creatividad fundamental para el arquitecto?

3) ¿Te consideras creativo?

4) ¿Enseñas a tus estudiantes un método de diseño?

5) ¿Existe alguna clase específica de creatividad?

6) ¿Realizas a tus estudiantes alguna prueba específica de creatividad?

7) ¿La escuela motiva expresamente a desarrollar la creatividad en los alumnos con alguna actividad extracurricular?

8) ¿Motivas expresamente la creatividad?

9) ¿La creatividad es innata?

10) ¿Consideras creativo dibujar y proyectar a mano?

11) ¿Enseñas a diseñar a mano?

12) ¿Permites diseñar directamente en la computadora?

13) ¿Diseñar en la computadora es creativo?

14) ¿Enseñas a diseñar a mano y dibujar en la computadora?

15) ¿Crees que los renders venden la arquitectura?

16) ¿Las tecnologías constructivas requieren de la creatividad?

17) ¿Las tecnologías digitales requieren de la creatividad?

18) ¿Construir requiere de la creatividad?

19) ¿La gestión requiere creatividad?

20) ¿Se requiere creatividad para elegir las estrategias de diseño? 


\section{Revista Iberoamericana para la Investigación y el Desarrollo Educativo ISSN $2007-7467$}

Figura 3. Encuesta a los profesores

\begin{tabular}{|c|c|c|c|c|c|c|c|c|c|c|c|c|c|c|c|c|c|c|c|c|}
\hline$\%$ & 1 & 2 & 3 & 4 & 5 & 6 & 7 & 8 & 9 & 10 & 11 & 12 & 13 & 14 & 15 & 16 & 17 & 18 & 19 & 20 \\
\hline $\begin{array}{l}10 \\
0\end{array}$ & & & & & & & & & & & & & & & & & & & & \\
\hline 95 & & & & & & & & & & & & & & & & & & & & \\
\hline 90 & & & & & & & & & & & & & & & & & & & & \\
\hline 85 & & & & & & & & & & & & & & & & & & & & \\
\hline 80 & & & & & & & & & & & & & & & & & & & & \\
\hline 75 & & & & & & & & & & & & & & & & & & & & \\
\hline 70 & & & & & & & & & & & & & & & & & & & & \\
\hline 65 & & & & & & & & & & & & & & & & & & & & \\
\hline 60 & & & & & & & & & & & & & & & & & & & & \\
\hline 55 & & & & & & & & & & & & & & & & & & & & \\
\hline 50 & & & & & & & & & & & & & & & & & & & & \\
\hline 45 & & & & & & & & & & & & & & & & & & & & \\
\hline 40 & & & & & & & & & & & & & & & & & & & & \\
\hline 35 & & & & & & & & & & & & & & & & & & & & \\
\hline 30 & & & & & & & & & & & & & & & & & & & & \\
\hline 25 & & & & & & & & & & & & & & & & & & & & \\
\hline 20 & & & & & & & & & & & & & & & & & & & & \\
\hline 15 & & & & & & & & & & & & & & & & & & & & \\
\hline 10 & & & & & & & & & & & & & & & & & & & & \\
\hline 5 & & & & & & & & & & & & & & & & & & & & \\
\hline 0 & & & & & & & & & & & & & & & & & & & & \\
\hline
\end{tabular}

Fuente: Elaboración propia

\section{Preguntas a los egresados}

1) ¿La arquitectura es un arte?

2) ¿Consideras la creatividad fundamental para el arquitecto?

3) ¿Te consideras creativo?

4) ¿Te enseñaron de estudiante un método de diseño?

5) ¿Recibiste alguna clase específica de creatividad?

6) ¿Te hicieron alguna prueba específica de creatividad?

7) ¿La escuela te motivó expresamente a desarrollar la creatividad en los alumnos con alguna actividad extracurricular?

8) ¿Motivas expresamente la creatividad?

9) ¿La creatividad es innata?

10) ¿Consideras creativo dibujar y proyectar a mano?

11) ¿Te enseñaron a diseñar a mano? 


\section{Revista Iberoamericana para la \\ Investigación y el Desarrollo Educativo \\ ISSN $2007-7467$}

12) ¿Te permitieron diseñar directamente en la computadora?

13) ¿Diseñar en la computadora es creativo?

14) ¿Te enseñaron a diseñar a mano y dibujar en la computadora?

15) ¿Crees que los renders venden la arquitectura?

16) ¿Las tecnologías constructivas requieren de la creatividad?

17) ¿Las tecnologías digitales requieren de la creatividad?

18) ¿Construir requiere de la creatividad?

19) ¿La gestión requiere creatividad?

20) ¿Se requiere creatividad para elegir las estrategias de diseño?

Figura 4. Encuesta a los egresados

\begin{tabular}{|c|c|c|c|c|c|c|c|c|c|c|c|c|c|c|c|c|c|c|c|c|}
\hline$\%$ & 1 & 2 & 3 & 4 & 5 & 6 & 7 & 8 & 9 & 10 & 11 & 12 & 13 & 14 & 15 & 16 & 17 & 18 & 19 & 20 \\
\hline $\begin{array}{l}10 \\
0\end{array}$ & & & & & & & & & & & & & & & & & & & & \\
\hline 95 & & & & & & & & & & & & & & & & & & & & \\
\hline 90 & & & & & & & & & & & & & & & & & & & & \\
\hline 85 & & & & & & & & & & & & & & & & & & & & \\
\hline 80 & & & & & & & & & & & & & & & & & & & & \\
\hline 75 & & & & & & & & & & & & & & & & & & & & \\
\hline 70 & & & & & & & & & & & & & & & & & & & & \\
\hline 65 & & & & & & & & & & & & & & & & & & & & \\
\hline 60 & & & & & & & & & & & & & & & & & & & & \\
\hline 55 & & & & & & & & & & & & & & & & & & & & \\
\hline 50 & & & & & & & & & & & & & & & & & & & & \\
\hline 45 & & & & & & & & & & & & & & & & & & & & \\
\hline 40 & & & & & & & & & & & & & & & & & & & & \\
\hline 35 & & & & & & & & & & & & & & & & & & & & \\
\hline 30 & & & & & & & & & & & & & & & & & & & & \\
\hline 25 & & & & & & & & & & & & & & & & & & & & \\
\hline 20 & & & & & & & & & & & & & & & & & & & & \\
\hline 15 & & & & & & & & & & & & & & & & & & & & \\
\hline 10 & & & & & & & & & & & & & & & & & & & & \\
\hline 5 & & & & & & & & & & & & & & & & & & & & \\
\hline 0 & & & & & & & & & & & & & & & & & & & & \\
\hline
\end{tabular}

Fuente: Elaboración propia 


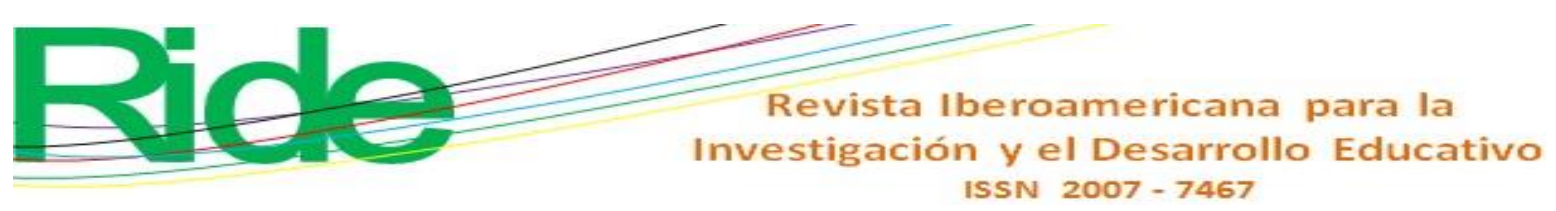

\section{Entrevistas}

Las entrevistas realizadas fueron semiestructuradas para que los entrevistados pudieran continuar desarrollando alguna idea resultado de alguno de los temas.

Las entrevistas se realizaron con base en las siguientes preguntas:

1) ¿El arquitecto debe ser creativo?

2) ¿La arquitectura sustentable requiere de la creatividad?

3) ¿El diseño de las diferentes instalaciones en una obra requiere de la creatividad?

4) ¿Debiera de estar la creatividad como indispensable en el perfil de ingreso?

5) ¿Debiera de estar la creatividad como indispensable en el perfil de egreso?

6) ¿Estás al tanto de que la Universidad de San Diego incluyó el estudio de la neuroarquitectura en su plan de estudios?

7) ¿Debieran promoverse cursos de neuroarquitectura en la universidad?

8) ¿Cuál sería en tu opinión la principal característica del arquitecto?

9) ¿Con la infraestructura y equipo existente es suficiente para desarrollar la creatividad de los estudiantes?

10) ¿La creatividad es una competencia específica en el plan de estudios?

11) ¿El nuevo plan de estudios de la carrera de Arquitectura qué perfil de egreso pretende de sus estudiantes?

12) ¿Cuál debiera ser la principal característica de un egresado de la carrera de Arquitectura?

13) ¿Por qué?

14) ¿La Escuela de Arquitectura cuenta con la infraestructura y mobiliario adecuados a las necesidades de enseñanza de la arquitectura, para lograr este fin?

15) ¿La Universidad de Sonora está dispuesta a apoyar a el Departamento de Arquitectura y Diseño para lograr que cuente con dichas instalaciones?

16) ¿Teniendo una mejor infraestructura y equipo ayudaría a desarrollar la creatividad de los estudiantes?

17) ¿Se da expresamente a los profesores las facilidades y las herramientas necesarias para su continua superación docente?

18) ¿Los profesores están dispuestos a superarse?

19) Si la respuesta es negativa, ¿por qué? 


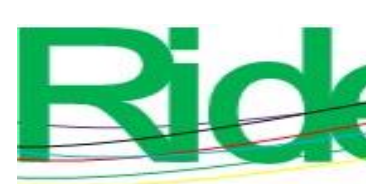

Revista Iberoamericana para la Investigación y el Desarrollo Educativo ISSN $2007-7467$

20) ¿Los profesores fomentan la creatividad en sus clases?

21) ¿La creatividad es importante en los alumnos de arquitectura?

22) ¿La creatividad es una competencia específica en el plan de estudios?

\section{Resultados}

Las entrevistas arrojaron datos diversos y preocupantes. Todos piensan que la creatividad es necesaria para el estudiante de arquitectura, sin embargo, no consideran necesario que esté expresamente señalada en el plan de estudios como una competencia a adquirir y desarrollar, y desafortunadamente un porcentaje mínimo pero importante considera que con el mobiliario y equipo con que se cuenta es suficiente para la correcta enseñanza de la arquitectura, cosa que desafortunadamente no es así. Ven a la creatividad como algo que deben de tener los alumnos, y si no la tienen, pueden suplirla con trabajo. Estos puntos de vistas no distinguen entre la creatividad específica dirigida a un arte como la arquitectura y la creatividad acumulada por la experiencia empírica.

Respecto a los espacios físicos para el estudio, el equipamiento necesario y el mobiliario adecuado, casi todos coinciden en que es indispensable contar con ellos. Esto nos lleva a pensar que necesitamos de estructuras modernas que ayuden a los alumnos a desarrollar el pensamiento creativo arquitectónico.

Ahora bien, el Ing. Heriberto Encinas, secretario de la División de Humanidades y Bellas Artes, opinó que deberá hacerse un cambio en la forma de enseñar la arquitectura, actualizarse para tener la competencia creativa para hacer una arquitectura innovadora, crear algo diferente pero que resuelva los problemas del presente con miras en el futuro. El doctor en Arquitectura Oscar Palafox, coordinador del Escuela de Arquitectura, comentó la importancia de la creatividad en los estudiantes, pero no juzga necesario poner como una competencia específica la creatividad. Además, expresó que con lo que actualmente se cuenta en infraestructura, servicios y mobiliario es suficiente, pero que si se considerara necesario adquirir otros elementos de infraestructura se debería presentar una propuesta fundamentada al Departamento y este gestionaría ante Rectoría para lograr obtener los recursos y la implementación de estos. Estos comentarios consideran a la creatividad como muy importante en los arquitectos, sin embargo, se sigue pensando que es un problema de los alumnos y no de la enseñanza, y no considera cómo ambientalmente en una escuela de arquitectura se puede contribuir con enseñar y aprender los aspectos fundamentales de la 


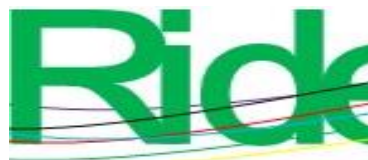

Revista Iberoamericana para la Investigación y el Desarrollo Educativo ISSN $2007-7467$

creatividad. El maestro en Arquitectura Luis Franco, docente de tiempo completo y fundador de la Escuela de Arquitectura y de Diseño, propone una fundamentación teórica de la creatividad para así tener elementos suficientes en qué sustentarla y con ello lograr mejores resultados en el quehacer arquitectónico.

Para el arquitecto Raúl Gutiérrez, profesor de la Escuela de Arquitectura, la creatividad es fundamental para que el estudiante esté en condiciones de realizarse profesionalmente como arquitecto. Según él, debería de estar presente en el plan de estudios de manera formal $\mathrm{y}$, por supuesto, preparar a la planta docente en neurociencias y neuroarquitectura para estar en condiciones de fomentar y acrecentar la competencia creativa. Estos comentarios son muy importantes, pues no se puede introducir la creatividad en el plan de estudios de Arquitectura si los maestros no están preparado y no tienen claro qué es la creatividad y su vínculo con la neurociencia. El arquitecto Fernando Padilla, profesionista independiente que trabaja en Guadalajara, Jalisco, comentó que en el ejercicio profesional es imprescindible la creatividad en todos los aspectos del quehacer arquitectónico, que sin la creatividad en todos los niveles de trabajo la arquitectura quedaría reducida a nada, seríamos solo diseñadores y constructores de espacios sin sentido personalizado, sin hacer ninguna aportación para cada uno de nuestros clientes, lo cual es lo que ellos verdaderamente necesitan: espacios propios y personales. Estos puntos de vistas coinciden totalmente con nuestra hipótesis acerca de la creatividad en la formación de los arquitectos.

\section{Discusiones}

En las encuestas se hicieron preguntas sobre la importancia de la creatividad y obtuvimos los siguientes resultados. En cuanto a las preguntas uno, dos y tres, la gran mayoría considera que la arquitectura es un arte, la creatividad es fundamental y sí se consideran creativos. Respecto a la pregunta cuatro, los estudiantes y los egresados consideran que sí les enseñaron un método para diseñar; los profesores todos expresaron haberlo enseñado. En la pregunta cinco, solo los maestros, en su mayoría, consideraron que existe una clase específica de creatividad. En la pregunta seis, sobre si se les había realizado alguna prueba específica de creatividad, la gran mayoría respondió negativamente. A la pregunta siete, donde se les cuestionó sobre si la escuela motiva específicamente la creatividad, $100 \%$ respondió negativamente. Mientras que respecto a la pregunta ocho, que indagó sobre si los profesores motivan la creatividad, solo estos consideraron que sí; los 


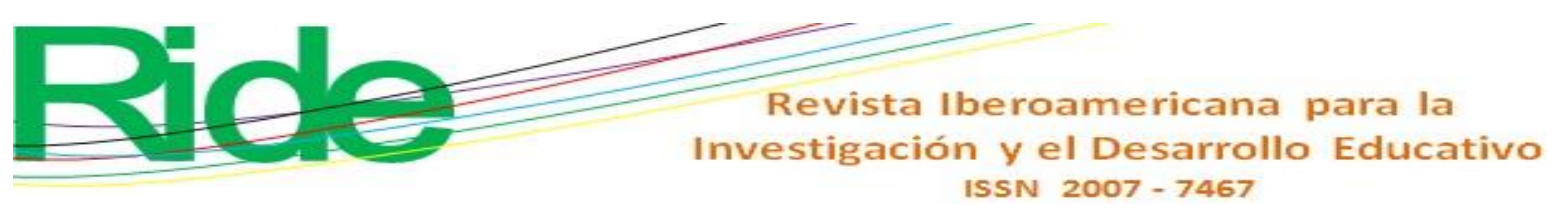

alumnos y los egresados respondieron negativamente. En la pregunta nueve se preguntó si consideraban innata a la creatividad, todos respondieron que no. En la pregunta 10, si consideraban creativo dibujar y diseñar a mano: todos respondieron afirmativamente. En la pregunta 11, sobre si se enseña a diseñar a mano, los profesores quedaron divididos y $65 \%$ de los estudiantes y egresados dijeron que no. En la pregunta 12, el porcentaje mayor de respuestas fue que se permite diseñar directamente en la computadora. Todos coincidieron que diseñar en la computadora es creativo (pregunta 13). En la pregunta 14, la mayoría coincidió en que no se les enseña a diseñar a mano y dibujar en la computadora. Respecto a la pregunta número 15 , sobre si los renders venden la arquitectura, solo los estudiantes en su mayoría respondieron que sí. En las preguntas 16, 17, 18, 19 y 20, la respuesta a todas ella fue que la creatividad es necesaria en todos los aspectos del trabajo arquitectónico, incluyendo la gestión, lo estructural, la elección de las estrategias de diseño, construir y el uso de las tecnologías de la construcción.

De acuerdo con los resultados de esta investigación, hay datos preocupantes que requieren un tratamiento particularizado para poder resolver el problema y sus causas. Los resultados obtenidos tanto de la observación como de la revisión del plan de estudios del 2018 y las encuestas y las entrevistas aclaran el panorama sobre la falta de inclusión específica de la creatividad en el espectro holístico como una competencia necesaria para el correcto desempeño de la creación arquitectónica. Si bien es cierto que en términos generales la creatividad se considera inherente al proceso metodológico del quehacer arquitectónico y dentro del plan de estudios existen asignaturas que ayudan a reforzarla y practicarla, se infiere como algo necesario pero inherente tanto en los estudiantes como en los profesionales de la arquitectura y no se juzga importante mencionarla ni darle demasiada importancia. Debido a esta situación, la creatividad ha sido dejada fuera de los requisitos de ingreso y de egreso, con lo que se ha minimizado el papel trascendental que tiene en el desarrollo del proyecto arquitectónico y la labor profesional del arquitecto.

Al revisar el nuevo plan de estudios se observó que solo en el microdiseño curricular se incluye a la creatividad como una habilidad requerida, pero ni en el modelo de actuación del profesional ni en los perfiles de ingreso y de egreso de los estudiantes, así como tampoco en las competencias profesionales y disciplinares, aparece de forma explícita y en el objetivo general igualmente no se contempla. Podemos observar cómo solo en el primer objetivo 


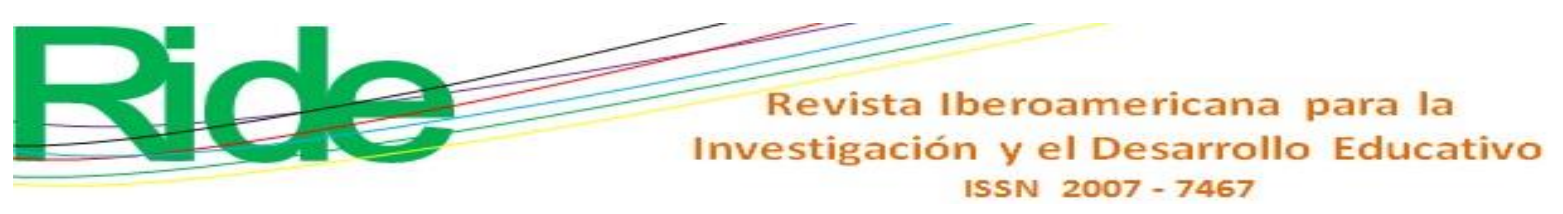

específico de la descripción de la carrera se menciona la promoción del pensamiento lógico, crítico y creativo (Universidad de Sonora, 2020).

De la revisión del microdiseño curricular del plan de estudios observamos que no existe ninguna asignatura dirigida exclusivamente al desarrollo del pensamiento creativo. Tampoco existen indicaciones metodológicas acerca de la contribución de las otras materias a la formación de la creatividad. No existe en el proceso de evaluación las indicaciones metodológicas para evaluar el desarrollo de la creatividad.

Al observar la infraestructura espacial, los salones dedicados a impartir los talleres, para comprobar si tenían los requerimientos espaciales, de mobiliario y equipo que necesitan para poder desarrollar las actividades de enseñanza aprendizaje, se detectó que niguna de las aulas donde se imparten las clases de los diferentes talleres reúne las condiciones óptimas necesarias para el aprendizaje, mucho menos existe un aula con simulaciones virtuales y equipamiento adecuado para el desarrollo del pensamiento creativo. Desafortunadamente, la infraestructura, el mobiliario, los servicios y las tecnologías son obsoletos e inadecuados para la enseñanza de la arquitectura y los espacios no contribuyen a lograr la concentración requerida y el trabajo creativo. Las aulas no tienen el mobiliario necesario, y el que existe no está en buenas condiciones para que los estudiantes puedan realizar su aprendizaje de forma correcta.

A través de observar cómo trabajan los estudiantes, hemos podido detectar errores en el proceso del diseño, generalmente sin un método, al tratar de diseñar utilizando la computadora como primera instancia. Eso se observó tanto en estudiantes como en jóvenes egresados. Existe en las nuevas generaciones la idea poco afortunada de que así debe ser, pero al hacerlo de esta manera se brincan algo sumamente importante, la metodología del diseño arquitectónico, porque esta nos ayuda a ordenar las etapas que debemos de realizar para tal fin. Cuando se utiliza directamente la computadora para diseñar se está olvidando que, si bien es una excelente herramienta de dibujo, no piensa, no diseña, solo gráfica. Solo con mucha experiencia se pudiera, si así se deseara, recurrir a esta estrategia, pero generalmente requiere de mucho más tiempo para lograr el resultado deseado.

Es común escuchar que los renders, que son las perspectivas realizadas por computadora, son los que "venden" la arquitectura. Pensar así implica que el proyecto es secundario, la creatividad espacial secundaria, y nada más equivocado, porque el objetivo de la arquitectura es la creatividad espacial. Los renders son una excelente herramienta para 


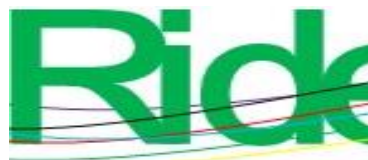

Revista Iberoamericana para la
Investigación y el Desarrollo Educativo
ISSN $2007-7467$

mostrar nuestro proyecto, pero deberán realizarse después de haber concluido con la etapa del diseño, para así poder mostrar de forma profesional nuestras ideas, nuestra propuesta: no confundamos el ser un excelente dibujante de renders con ser un excelente arquitecto. El proceso del diseño arquitectónico inicia en la mente humana, continúa a mano y termina con el apoyo de la tecnología, que mucho nos ayuda en esta última etapa de graficación.

Observamos mediante la visita a las aulas de trabajo que algunos profesores confunden creatividad con una ordenación de espacios que aparentemente funciona, y cuando esto sucede el resultado es una propuesta en donde, en el mejor de los casos, se incluye todo el listado de necesidades, pero sin una concepción espacial adecuada, que brinde, además de la utilidad, las sensaciones que hacen que sea más agradable realizar la actividad para la que fue diseñado.

En el proceso de la enseñanza se le da mucha importancia al funcionamiento, a la forma y a lo estético, tres factores de suma importancia que forman parte de la arquitectura, pero se olvida de la creatividad para lograr las sensaciones apropiadas y el papel fundamental que tiene en el proceso del diseño, tanto en el diseño estructural, de las instalaciones, como en las estrategias de diseño, en la elección de los materiales adecuados, de las tecnologías y de los procesos constructivos, todo esto requiere de la creatividad, poco requerida directamente en las aula; pareciera que se supone que va implícita, y no es así, por eso debemos hacer un énfasis especial en este punto, porque es la base del diseño.

Apoyados en las reflexiones anteriores, los docentes debemos saber guiar a los alumnos en el camino maravilloso de la creación arquitectónica, en el eureka de la creación, cuando lo que imaginamos se convierte en realidad mediante la graficación de dicha creación, orientando al estudiante, proporcionándole la metodología necesaria para este fin, durante todo el proceso metodológico del diseño arquitectónico, tomando conciencia de que la máquina modelará nuestra propuesta arquitectónica; cuando lo que imaginamos lo podamos visualizar mediante los diferentes planos arquitectónicos, que nos servirán para poder llevar a cabo la construcción real de los espacios diseñados.

Los estudiantes en general utilizan una metodología básica con algunas deficiencias, que habrá que mejorar. Al analizar sus propuestas arquitectónicas, una gran mayoría se basa en una propuesta formal atractiva, lo cual no tiene nada de malo; el problema es que descuidan el aspecto creativo espacial-sensorial, y en algunos de los casos el aspecto formal no corresponde a los planos presentados. También he observado en los concursos en los que 


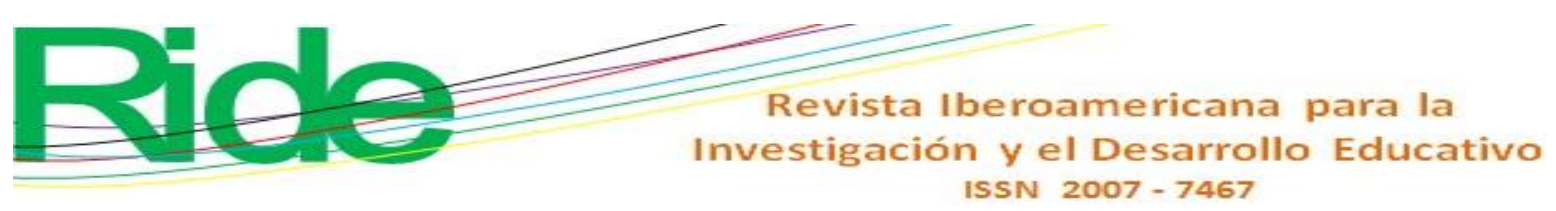

he participado como jurado de obra arquitectónica, tanto de alumnos que representaron a la Universidad de Sonora como de jóvenes arquitectos que presentan su ópera prima, que sucede lo mismo, el uso indiscriminado de los softwares tratando, tal vez de forma inadvertida, de suplir la creatividad con solo una presentación atractiva.

\section{Propuesta general}

El resultado de este estudio arrojó datos preocupantes y para solucionar esta situación se propone la aplicación de una metodología epistemológica que ayude a resolver la problemática existente tanto en el macro como en el microdiseño curricular, y que contemple incluir la creatividad como una competencia necesaria, no solo enunciarla, sino incluirla en el perfil de ingreso de los aspirantes, y que enfatice que durante el proceso de aprendizaje es necesario desarrollar la competencia creativa, indispensable en el perfil de egreso de los futuros arquitectos.

\section{Propuestas específicas}

1) Incorporar en el macrodiseño curricular la creatividad como parte del objetivo principal.

2) Incluir en el microdiseño curricular la creatividad como eje rector de todas las asignaturas, incluidas las teóricas.

3) Agregar al microdiseño curricular una asignatura de neuroarquitectura.

4) Agregar la creatividad en el perfil de ingreso y la competencia creativa como indispensable en los estudiantes egresados.

5) La realización de pruebas de creatividad en todas las asignaturas y todos los semestres de la carrera para poder evaluar el avance adquirido semestralmente.

6) Capacitar a la planta de docente en la creatividad para poder detectarla, evaluarla y promoverla.

7) Añadir cursos periódicos de neurociencias y neuroarquitectura para la capacitación de los maestros.

8) Contratar expertos en estos temas o capacitar a algunos maestros para la impartición de los cursos, seminarios y diplomados. 


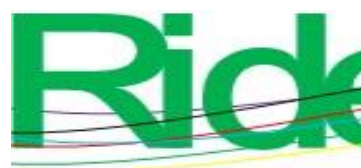

Revista Iberoamericana para la Investigación y el Desarrollo Educativo ISSN $2007-7467$

9) La inclusión de las nuevas tecnologías en la aplicación de la creatividad para adquirir la competencia creativa dentro de un momento histórico muy especial, apoyados en el pasado, resolviendo los problemas presentes con una visión hacia el futuro.

10) La creación de un centro de entrenamiento digital, donde se capacite tanto a los estudiantes como a los profesores en el uso de las tecnologías digitales, que no sea parte del programa curricular, sino que sea un centro de capacitación y superación constante.

La inclusión de las tecnologías será de gran importancia porque para los estudiantes del siglo XXI es indispensable que utilicen las nuevas herramientas digitales para mostrar su creatividad. Pero requieren de capacitación constante, ya que es común la aparición de nuevos softwares, de ahí la importancia de un centro de entrenamiento tecnológico

Es sumamente importante, de igual forma, realizar pruebas de creatividad a los estudiantes, porque con los resultados podremos valorar el desarrollo personal de esta competencia. Una de las pruebas de pensamiento creativo que constituye un método de referencia para medir la creatividad es la de Torrance (1977), una prueba verbal en la que se les pide a los participantes que a objetos comunes les den usos inusuales; también existe una prueba figurativa donde se pide incorporar formas simples o abstractas en un dibujo complejo (Thorne, 2008). Los criterios de evaluación de las respuestas nos servirán de referencia para estimular los procesos creativos. Según Thorne (2008), dicho criterios son los siguientes:

- $\quad$ Fluidez: si se tienen muchas ideas.

- $\quad$ Flexibilidad: si se piensan diferentes formas de proceder.

- $\quad$ Originalidad: si se piensan aspectos únicos.

- $\quad$ Elaboración: si se piensan complementos a la idea que se ha tenido.

- $\quad$ Ejecución: si se logra implementar en la práctica la creatividad.

Existen varias pruebas que pueden ayudarnos para evaluar la creatividad de los estudiantes, por ejemplo, el ya mencionado test de Torrance (1977), el test de Guilford (1949) y el de Wallach y Kogan (1965). 


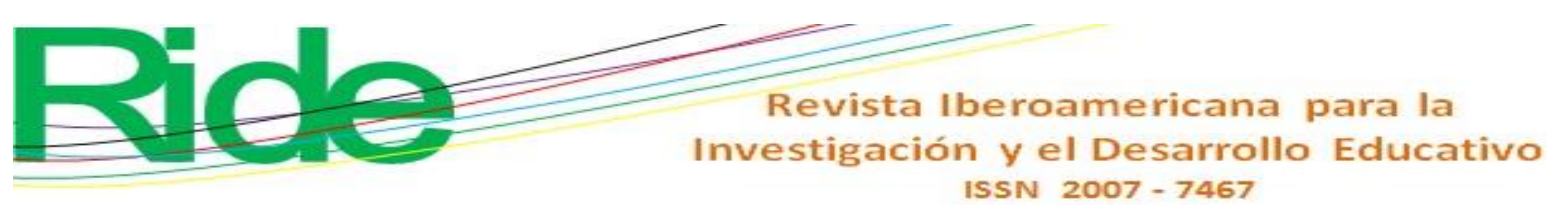

\section{Conclusiones}

Después de una minuciosa revisión de los resultados de esta investigación, se concluye que tenemos un problema en la manera en que hemos estado educando a nuestros estudiantes, y para poder resolverlo deberemos aportar una nueva visión que complemente lo que actualmente tenemos y que nos permita ofrecerles una mejor educación para dotarlos de una preparación adecuada que los ayude a enfrentar su práctica profesional. Debemos reconocer a la creatividad como parte intrínseca del diseño arquitectónico, fomentarla, practicarla, mejorarla, y para lograr esto se requiere que el plan de estudios enfatice en su importancia, reforzar las asignaturas que la promueven y la requieren para que tanto las autoridades como los profesores y los estudiantes la reconozcan, la ejerciten y la utilicen conscientemente en los proyectos arquitectónicos. Se concluye, también, que es necesario dotar a la Escuela de Arquitectura de espacios, infraestructura, tecnología y servicios necesarios y de actualidad; reforzar la competencia creativa, el estudio, actualización y dominio de las tecnologías tanto de los profesores como de los estudiantes y de una correcta labor de seguimiento y perfeccionamiento del plan de estudios, así como del importante trabajo que las academias deben hacer en el seguimiento, revisión y aportación para el mejoramiento del proceso educativo.

Hay que enfatizar que la creatividad no es exclusiva del aspecto formal-estético, del proceso metodológico de diseñar la arquitectura, la creatividad también deberá aplicarse en los aspectos tecnológicos, constructivos, en la utilización de los materiales adecuados y en la aplicación de estrategias de diseño apropiadas que ayuden a lograr una propuesta proyectualespacial-sensorial adecuada, funcional y única.

Se deberá reconocer dónde se origina el estado de la deficiencia metodológica y cómo está afectando a los estudiantes y futuros arquitectos en la creatividad de su trabajo profesional. Es muy importante capacitar a la planta académica y promover un cambio de mentalidad, de ser necesario. Ver el pasado para adecuar lo importante y necesario a la nueva época, en un momento distinto con necesidades diferentes y grandes oportunidades y sobre todo la inclusión de nuevas tecnologías y formas de pensamiento prevalecientes en la actualidad para poder preparar profesionales competentes no solo en el presente sino también en el futuro. 


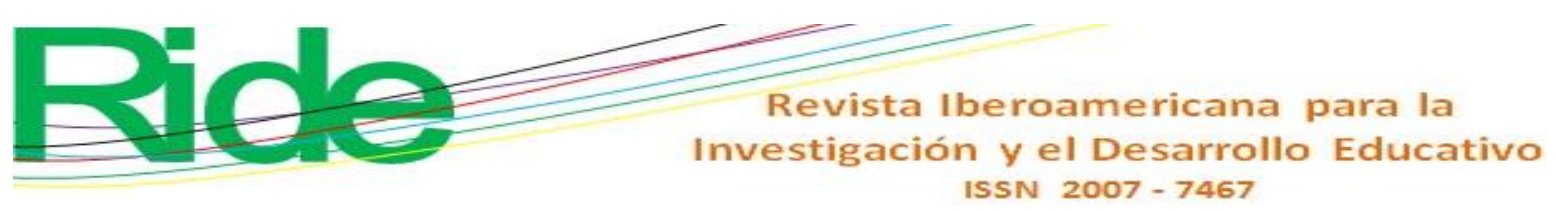

\section{Referencias}

Alcaraz, E. A. (2006). El emprendedor de éxito. Ciudad de México, México: McGrawHill/Interamericana.

Ando, T., Heneghan, T. y Pare, R. (1996). Tadao Ando: The Colours of Light. New York, United States: Phaidon.

Bonilla, I. (s. f.). ¿Qué es arquitectura? Definiciones de arquitectura por arquitectos famosos. ¿Cuál es la diferencia entre arquitectura, escultura y construcción? Recuperado de https://www.academia.edu/2916413/Qu\%C3\%A9_es_arquitectura_una_definici\%C 3\%B3n_contrastada_con_otras_de_famosos_arquitectos.

Cerda, H. (2000). La creatividad en la ciencia y en la educación. Bogotá, Colombia: Editorial Magisterio.

Corral, V. y Díaz, X. (2009). Factores asociados a la reprobación de los estudiantes de la Universidad de Sonora. Ponencia presentada en el X Congreso Nacional De Investigación Educativa. Veracruz, del 21 al 25 de septiembre de 2009.

De la Barrera, M. L., y Donolo, D. S. (2009). Neurociencias y su importancia en contextos de aprendizaje. Revista Digital Universitaria, 10(4). Recuperado de http://www.revista.unam.mx/vol.10/num4/art20/art20.pdf.

Elizondo, A. M. y Rivera, N. L. (2017). El espacio físico y la mente: Reflexión sobre la neuroarquitectura. Cuadernos de Arquitectura, 7(7), 41-47.

Esquivias, M. T. (2004). Creatividad: definiciones, antecedentes y aportaciones. Revista Digital Universitaria, 5(1). Recuperado de https://www.revista.unam.mx/vol.5/num1/art4/ene_art4.pdf.

Franch, E. B. y Camacho, M. M. (2016). El desarrollo de las ciudades desde la publicidad exterior y las neurociencias. Opción, 32(7), 231-247.

Guilford, J. P. (1949). The Guilford-Zimmerman Temperament Survey. Beverly Hills, United States: Sheridan Psychological Services.

Gutiérrez, L. (2017). Neuroarquitectura, creatividad y aprendizaje en el diseño arquitectónico. Paideia XXI, 6(7), 171-189.

Huerta, J. M. (16 de febrero de 2016). Pensamiento crítico, pensamiento analítico y pensamiento creativo. José Huerta. Gestión en TI. Recupeardo de https://josehuerta.es/gestion/personas/pensamiento-critico-pensamiento-analitico-ypensamiento-creativo. 


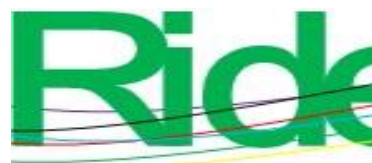

Revista Iberoamericana para la Investigación y el Desarrollo Educativo ISSN 2007 - 7467

Jung, M., Bowden, E. M., Haberman, J., Frymiare, J. L., Arambel, S., Greenblatt, R., Reber, P. J. and Kounios, J. (2004). Neural activity when people solve verbal problems with insight. PLoS Biology, 2(4).

Kolb, B. y Whishaw, I. Q. (2006). Neuropsicología humana. Ciudad de México, México: Médica Panamericana.

López de Asiain, M. (2005). "La formación medioambiental del arquitecto". Hacia un programa de docencia basado en la arquitectura y el medioambiente. (tesis doctoral). Universitat Politècnica de Catalunya, Cataluña. Recuperado de https://upcommons.upc.edu/handle/2117/93431.

McLaren, P., Huerta, L. M. y Rodríguez, M. G. (2010). El cambio educativo, el capitalismo global y la pedagogía crítica revolucionaria. Revista Mexicana de Investigación Educativa, 15, 1124-1130.

Mendoza, L., Zacarías, P. y Moreno, A. M. (2015). Investigación, docencia y vinculación social para el aprendizaje significativo de la arquitectura. Arquitecturas del Sur, 33(48), 56-67.

Portellano, J. A. (2000). Introducción a la neuropsicología. España: McGraw-Hill.

Punset, E. (s. f.). Neuroarquitectura: el reflejo por fuera de lo que somos por dentro. Diseño. Vip. Recuperado de https://xn--diseo-rta.vip/neuroarquitectura-el-reflejo-por-fuera/.

Rueda, G. (2014). Donde la arquitectura habita. Blucher Design Proceedings, 1(8), 259-261.

Silvia, P. J. (2008). Creativity and intelligence revisited: A latent variable analysis of Wallach and Kogan. Creativity Research Journal, 20(1), 34-39.

Terán, J. A. (2004). Consideraciones que deben tenerse en cuenta para la restauración arquitectónica. Conserva, (8), 102-122.

Thorne, K. (2008). Motivación y creatividad en clase. Barcelona, España: Grao.

Tirapu, J. y Luna, P. (2008). Neuropsicología de las funciones ejecutivas. En Manual de neuropsicología (pp. 219-259). España: Viguera Editores.

Torrance, E. P. (1977). Educación y capacidad creativa. Madrid, España: Marova.

Universidad de Sonora. (2018). Plan de estudios. Licenciatura de Arquitectura. Sonora, México: Universidad de Sonora. Recuperado de http://www.ofertaeducativa.uson.mx/wp-content/uploads/2019/03/PE-ARQ.pdf. 
